\title{
SARS-CoV-2 peritoneal positivity and emergency surgery: is there any putative predisposing factor?
}

\author{
Mirko Barone ${ }^{1,3}$ (D) $\cdot$ Massimo Ippoliti ${ }^{1} \cdot$ Felice Mucilli ${ }^{1,2}$
}

Received: 16 April 2021 / Accepted: 21 April 2021 / Published online: 3 May 2021

(C) Italian Society of Surgery (SIC) 2021

\section{Dear Editor,}

Severe acute respiratory syndrome coronavirus 2 (SARSCoV-2), causing coronavirus disease 2019 (COVID-19), has been declared a global pandemic. Belonging to betacoronavirus species, infection causes a spectrum of potentially life-threatening symptoms, such as acute distress respiratory disease. Moreover, COVID-2019 patients have often to struggle with also severe gastrointestinal complications, including ischemia [1]. SARS-CoV-2 RNA has been detected in several infected tissues, including peritoneal fluid [2]. However, its clinical relevance as far as pathophysiological mechanisms are still unclear. Although viral tropism for angiotensin-converting enzyme 2 (ACE 2 ) has been clearly demonstrated, as a target domain for serine protease coupled cell entry mechanism, to this date, no clear evidences about ACE2 peritoneal expression are available. Since the SARS-COV-2 Spike (S) glycoprotein complex processed by the cellular protease TMPRSS2 represents a critical step in the suppression of the modulatory activity of ACE2, the subsequent predominance of the ACE/AngII/AT1R axis and reduction of the protective function of the Ang (1-7)-Mas complex could result into a cytokine-bradykinin inflammatory response, Fas-induced apoptosis and oxidative damage leading to mucosal disruption [3]. Furthermore, the imbalance of the Angiotensin II could cause a self-sustained inflammatory state due to synergic action on the ERK1/2

Mirko Barone

mir87mb@libero.it

1 Department of General and Thoracic Surgery, SS. Annunziata Hospital, Chieti, Italy

2 University Department of Medical, Oral and Biotechnological Sciences, Gabriele d'Annunzio University of Chieti-Pescara, Chieti, Italy

3 Department of General and Oncological Surgery, SS. Annunziata Hospital, Via dei Vestini n.1, 66100 Chieti, Italy and p38 MAPK [4] with induction of proinflammatory cytokin gene expression as well as other soluble inflammatory mediators, such as the activation of the innate immune system tool-like-receptors (TLRs).

According to the need for ACE2-TMPRSS2 co-transport, the action of SARS-COV-2 is preferentially on tissues with high gene expressions. Target tissue, as reported by Zou et al. [5], in a scRNA-seq data analysis, are located specific cell lines into lung, heart, oesophagus, kidney, bladder and ileum; while it is not known whether a direct expression of ACE2 exists on peritoneal mesothelial cells. Regarding the gastrointestinal tract, the potential role of the gut in COVID19 infection has been amply demonstrated [6] with a stool virionic RNA positivity ranging between 20 and $47 \%$ of cases. Surprisingly, SARS-COV-2 faecal shedding seems to be prolonged, persisting up to several weeks after respiratory swab negativisation [7]. It is still unclear whether a prolonged positivity at the faecal samples correlates with a prolonged infectivity, although long-standing immunoreaction has been described in paucisymptomatic young patients with uneventful courses. The expression of ACE2 and its transmembrane serine protease are not homogeneous in the gastrointestinal tract. In this setting, a putative theory that would justify peritoneal contamination from SARS-COV-2 could be the effects of a direct cellular damage as far as of an increased capillary permeability. The breakdown of the intestinal barrier could, therefore, be the primum movens of extraluminal translocation. However, the coexistence of an impaired host's responses remains a crucial aspect with disruption of enterocytic tight junctions and cryptic $\mathrm{M}$ cells. An inflammatory and ischemic microvascular induced dysbiosis could therefore justify the SARS-COV-2 contamination in the peritoneal fluid.

To this date, only case reports and a small series of patients are reported in the literature and are characterised by discrepancy of evidences. Emergency procedures as far as the heterogeneity of patients could justify the dissimilarity of results. Coccolini et al. [2], first, reported a case of 
peritoneal RT-PCR positivity in a surgically treated COVID19 patient due an uncomplicated ileal volvulus. Adopting a nucleotide assay protocol based on the amplification of three viral targets (SARS-COV-2 RdRP, N, E), Authors identified the presence of a significantly higher peritoneal viral load compared to the upper airways. Similarly, Barberis et al. [8], in a 71-year-old woman who underwent subtotal intra-abdominal colectomy for severe colitis, confirmed the virionic presence in the peritoneal swab. Rimini et al. [9] showed an amplified RT-PCR positivity in one case of exploratory laparotomy and small bowel resection due to an incarcerated umbilical hernia with concomitant loop necrosis. On the other hand, both Negasering et al. [10] and Vudayagiri et al. [11] did not find any intraperitoneal molecular positivity approaching cases of laparoscopic appendectomies in COVID-19 patients. In support of such evidence, a small case series of five patients, where the Authors [12] did not detect immunostaining in gene amplification assays. According to the scarce statistical representativeness of the available cases, exhaustive conclusions and evidences are far to be drawn. Despite this, some trends worthy of reflection emerge from the analysis of data. In fact, 50\% (2/4) of patients with preoperative findings (radiological and endoscopic) of ischemia and/or indirect signs of microvascular injury (ulceration, bleeding) had positive peritoneal swabs. In contrast, only $16.67 \%$ (n.1/6) of patients without preoperative signs of ischemia showed RT-PCR SARS-CoV-2 abdominal positivity. The ischemic insult was associated with a threefold increased relative risk of peritoneal fluid positivity albeit in the absence of a relevant statistical correlation (RR 3.00 [95\%CI 0.39-23.07; $p=0.29$ ]). Although strong evidences are lacking, from a preliminary examination it would seem the peritoneal involvement in COVID19 is mainly attributable to a secondary visceral outbreak rather than to a primitive serosal injury. The small cohort of patients, absolutely not representative, does not allow us to formulate further hypotheses that can refute or not what emerged. Surely, if the data were confirmed, COVID-19 surgical patients could be clustered into two scenarios: cases with preoperative or clinical findings suspected of visceral ischemia (at risk of peritoneal positivity) and patients with no findings of vascular organ insufficiency. In clinical practice this would entail significant implications as, for patients at risk, it would be necessary to strictly avoid minimally invasive surgical approaches and thus the risk of air-borne infections; while, for the second cohort, indications of a minimally invasive approach could be pursued by preserving the safety of healthcare professionals and the operating environment in general. Multicentric studies are urgently needed which can provide comprehensive answers in the immediate future.
Author contributions All the authors contributed to this paper, to the overall concept and outline of the manuscript and to the discussion and design of the manuscript as far as the review of literature.

Funding None declared.

\section{Declarations}

Conflicts of interest The authors have no conflicts of interest to declare.

Ethical approval The authors are accountable for all aspects of the work in ensuring that questions related to the accuracy or integrity of any part of the work are appropriately investigated and resolved.

\section{References}

1. Chen N, Zhou M, Dong X, Qu J, Gong F, Han Y, Qiu Y, Wang J, Liu Y, Wei Y, Xia J, Yu T, Zhang X, Zhang L (2020) Epidemiological and clinical characteristics of 99 cases of 2019 novel coronavirus pneumonia in Wuhan, China: a descriptive study. Lancet 395(10223):507-513

2. Coccolini F, Tartaglia D, Puglisi A, Giordano C, Pistello M, Lodato M, Chiarugi M (2020) SARS-CoV-2 is present in peritoneal fluid in COVID-19 patients. Ann Surg 272(3):e240-e242

3. Barone M, Ucciferri C, Cipollone G, Mucilli F (2020) Recombinant human angiotensin-converting enzyme 2 and COVID19 acute respiratory distress syndrome: a theoretical or a real resource? EJMO 4(2):139-140

4. Kiribayashi K, Masaki T, Naito T, Ogawa T, Ito T, Yorioka N, Kohno N (2005) Angiotensin II induces fibronectin expression in human peritoneal mesothelial cells via ERK1/2 and p38 MAPK. Kidney Int 67(3):1126-1135

5. Zou X, Chen K, Zou J, Han P, Hao J, Han Z (2020) Single-cell RNA-seq data analysis on the receptor ACE2 expression reveals the potential risk of different human organs vulnerable to 2019nCoV infection. Front Med 14(2):185-192

6. Xiao F, Tang M, Zheng X, Liu Y, Li X, Shan H (2020) Evidence for Gastrointestinal Infection of SARS-CoV-2. Gastroenterology 158(6):1831-1833.e3

7. Wu Y, Guo C, Tang L, Hong Z, Zhou J, Dong X, Yin H, Xiao Q, Tang Y, Qu X, Kuang L, Fang X, Mishra N, Lu J, Shan H, Jiang G, Huang X (2020) Prolonged presence of SARS-CoV-2 viral RNA in faecal samples. Lancet Gastroenterol Hepatol 5(5):434-435

8. Barberis A, Rutigliani M, Belli F, Ciferri E, Mori M, Filauro M. SARS-Cov-2 in peritoneal fluid: an important finding in the Covid-19 pandemic. Br J Surg. 2020. Doi: https://doi.org/10. 1002/bjs. 11816.

9. Rimini E, Atzori G, Viotti A et al. COVID-19 in the peritoneal fluid: does this evidence oblige to introduce new rules? Presentation of a Case Report, 12 May 2020, [Preprint -available at Research Square https://doi.org/https://doi.org/10.21203/rs.3. rs-28579/v1]

10. Ngaserin SH, Koh FH, Ong BC, Chew MH (2020) COVID-19 not detected in peritoneal fluid: a case of laparoscopic appendicectomy for acute appendicitis in a COVID-19-infected patient. Langenbecks Arch Surg 405(3):353-355

11. Vudayagiri L, Gusz J (2020) COVID-19 positive in nasopharyngeal swab but negative in peritoneal fluid: case report of perforated appendicitis. Cureus 12(7):e9412 
12. Seeliger B, Philouze G, Benotmane I, Mutter D, Pessaux P (2020) Is the severe acute respiratory syndrome coronavirus 2 (SARS-CoV-2) present intraperitoneally in patients with coronavirus disease 2019 (COVID-19) infection undergoing emergency operations? Surgery 168(2):220-221
Publisher's Note Springer Nature remains neutral with regard to jurisdictional claims in published maps and institutional affiliations. 